\title{
Mecanismos de adaptación a sequía en caupí (Vigna unguiculata (L.) Walp.). Una revisión
}

\section{Drought adaptation mechanisms in the cowpea (Vigna unguiculata (L.)Walp.). A review}

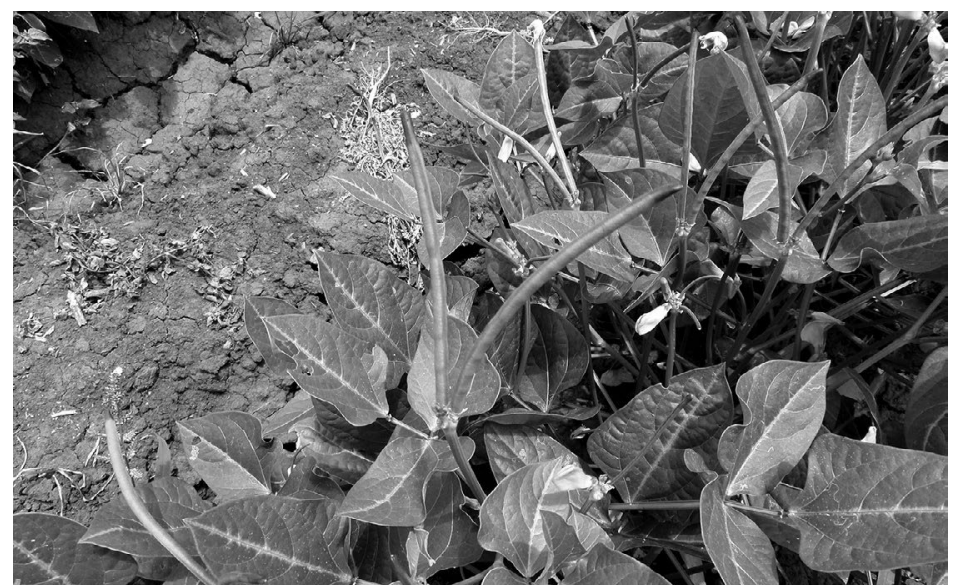

CARLOS CARDONA-AYALA $1,2,3$

ALFREDO JARMA-OROZCO

HERMES ARAMÉNDIZ-TATIS ${ }^{1}$

Caupí tolerante a sequía.

Foto: C.E Cardona-Ayala

\section{RESUMEN}

Este artículo presenta una revisión de los mecanismos de adaptación a sequía observados en fríjol caupí, mostrando las respuestas morfológicas, las relaciones hídricas e intercambio gaseoso, el ajuste osmótico, el sistema antioxidante y la actividad molecular. Se describen algunos indicadores que permiten la aproximación a un diagnóstico del nivel de estrés de las plantas tales como la relación raíz/parte aérea, densidad y profundidad de raíces, materia seca radical, área foliar específica, número de hojas y flores, senescencia foliar y abscisión foliar, componentes del rendimiento, conductancia estomática, transpiración, eficiencia en el uso del agua, contenido de osmolitos: prolina, glicina betaína y azucares; actividad de enzimas antioxidantes involucradas en procesos de detoxificación de las especies reactivas de oxígeno: catalasa, ascorbato peroxidasa, superóxido dismutasa y glutatión reductasa, así como la visualización de la frontera del conocimiento en este aspecto de gran importancia para el mejoramiento de la especie y los avances en biología molecular.

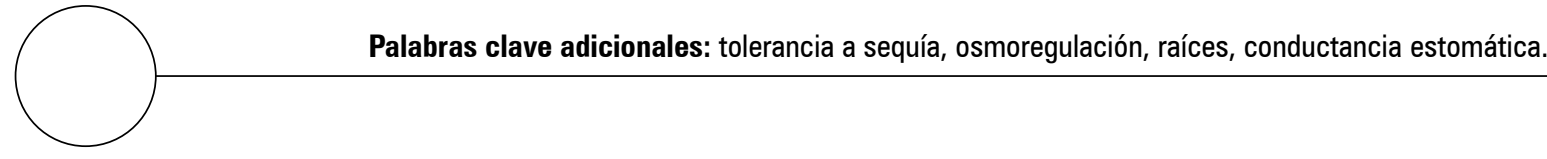

Facultad de Ciencias Agrícolas, Universidad de Córdoba, Montería (Colombia).

Doctorado en Ciencias Naturales para el Desarrollo, Instituto Tecnológico de Costa Rica, Cartago (Costa Rica).

Autor para correspondencia. ccardonaayala@yahoo.com. 


\section{ABSTRACT}

This article presents a review of the drought adaptation mechanisms observed in the cowpea bean, providing the morphological responses, water and gas exchange ratios, osmotic adjustment, antioxidant system and molecular activity. Furthermore, the indicators that allow for the diagnosis of the plant stress level are described, such as the root / shoot ratio, density and root depth, root dry-matter, specific leaf area, number of leaves and flowers, leaf senescence and abscission, yield components, stomatal conductance, transpiration, water-use efficiency, contents of the osmolytes: proline, glycine-betaine and sugars; activity of antioxidant enzymes involved in the detoxification processes of the oxygen-reactive species: catalase, ascorbate peroxidase, superoxide dismutase and glutathione reductase, as well as the visualization of the knowledge-frontiers in this area that are of great importance to the improvement of the species and advances in molecular biology.

Additional key words: drought tolerance, osmoregulation, roots, stomatal conductance.

Fecha de recepción: 01-09-2013

Aprobado para publicación: 31-10-2013
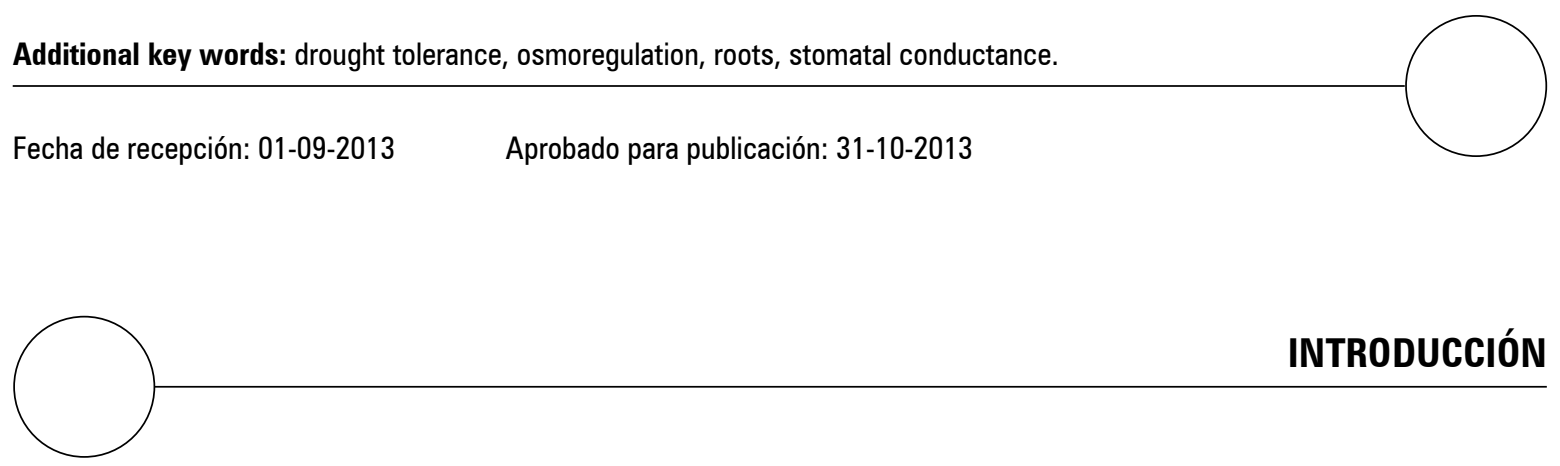

INTRODUCCIÓN

El frijol caupí es una leguminosa muy importante (Fabaceae) usada como alimento y forraje en las sabanas semi-áridas tropicales, debido a su tolerancia a sequía y su capacidad para crecer en suelos de baja fertilidad y es, a la vez, un cultivo valioso para los agricultores de muchas regiones del mundo (Timko et al., 2008); sin embargo se presenta baja productividad, que se atribuye al uso de cultivares de bajo rendimiento sumado al ataque de plagas y enfermedades, y a la carencia de genotipos mejor adaptados a sequía; en esta especie, el déficit hídrico reduce la conductancia estomática, el potencial hídrico foliar y la productividad de granos, presentándose amplia variabilidad en tolerancia a sequía de acuerdo con el grado de déficit hídrico (DoNascimento, 2009).

En las regiones semiáridas de África, la frecuencia e intensidad de las sequías han aumentado en los últimos 30 años (Hall et al., 2003) debido al cambio climático, evidenciado en el número de días con precipitación y cantidad de precipitación por día (Wahid et al., 2007). Esto ha pro- vocado un cambio a cultivos más tolerantes a la sequía como es el caso del caupí (Van Duivenbooden et al., 2002).

El conocimiento sobre los procesos y mecanismos de tolerancia a factores de estrés de tipo abiótico (sequía) y los efectos perjudiciales en especies cultivadas, particularmente en aquellas sometidas a condiciones secas, ayudará a mejorar su comportamiento agronómico mediante la incorporación de características relacionadas con tolerancia a sequía en los nuevos cultivares (Singh y Reddy, 2011; Beebe et al., 2013)

Considerando la gran importancia del fríjol caupí en la seguridad alimentaria en países tropicales y subtropicales en vía de desarrollo, se presenta esta revisión con el objetivo de visualizar los avances más recientes de los mecanismos y estrategias defensivas que presenta esta especie al ser sometida a déficit hídrico, hoy con más relevancia ante el impacto negativo en la agricultura del cambio climático (Nelson et al., 2009). 


\section{MODIFICACIONES DEL CRECIMIENTO Y DESARROLLO DE LA PLANTA}

Se ha observado que los mecanismos de tolerancia a sequía en caupí están fuertemente relacionados con el sistema radical. Así pues, densidad de raíces, profundidad de enraizamiento y materia seca radical por unidad de área, son parámetros que pueden ser utilizados como criterios de tolerancia a sequía, junto con el área foliar específica (Matsui y Singh, 2003).

En este mismo sentido, Polanía et al. (2012), en fríjol común (Phaseolus vulgaris L.) mostraron que la longitud de la raíz está correlacionada positivamente con la producción de biomasa aérea, de tal manera que los genotipos que presentan mayor profundidad radical están mejor adaptados a periodos de sequía prolongada, puesto que pueden explorar un mayor volumen de suelo $y$ extraer agua de perfiles inferiores.

Por las mismas razones, la relación raíz/parte aérea es también una característica importante en estudios sobre adaptación a sequía, ya que la absorción de agua puede ser mejorada a través de un sistema radical más extenso y profundo (Reis y Hall, 1987).

La altura de planta, el número de hojas y el número de flores por planta, disminuyen en la medida en que se agota el agua aprovechable del suelo. Además, con altos niveles de estrés hídrico se incrementan la senescencia y la abscisión de las hojas (Abayome y Abidoye, 2009; Ichi et al., 2013); también se reduce el tamaño de las hojas $y$, por consiguiente, el área foliar y el peso seco de la parte aérea, incluyendo las vainas (Qasem y Biftu, 2010; Okon, 2013).

La etapa reproductiva es la más sensible al déficit hídrico (Ahmed y Suliman, 2010); ya que se ven afectados algunos de los componentes del rendimiento (Ishiyaku y Aliyu, 2013). Estos autores encontraron que el número de vainas y el número de semillas por planta se vieron afectados, $y$, por consiguiente, el rendimiento de grano.
El escape a sequía representado en precocidad en días a floración y maduración ha sido usado para desarrollar cultivares en regiones donde las lluvias escasean; sin embargo, cuando tales variedades son expuestas a sequía intermitente en las etapas vegetativas o reproductivas, los rendimientos disminuyen significativamente (Agbicodo et al., 2009). Así mismo, la evitación a sequía se relaciona con el mantenimiento de altos potenciales hídricos en los tejidos de la planta, a pesar de la escasez de agua en el suelo, lo que obedece a una mayor profundización de las raíces, incremento de la conductividad hidráulica, disminución de la conductancia estomática, movimiento foliar para disminuir la absorción de luz y disminución del área foliar. La tolerancia a sequía se atribuye al ajuste osmótico, incremento en la elasticidad celular, disminución del tamaño de las células y resistencia del protoplasma a la desecación (Beebe et al., 2013).

Un buen indicador de tolerancia a sequía es la senescencia foliar tardía, que puede evaluarse en etapa juvenil, junto con otras variables, utilizando el método de la caja de madera (Agbicodo et al., 2009).

La senescencia foliar tardía concede resistencia a sequía, puesto que permite la recuperación postsequía y un segundo flujo de vainas compensa el bajo rendimiento del primero (Hall, 2004). También, la marchitez es el signo más común de estrés hídrico en plantas y puede evaluarse como índice de marchitez foliar, con la proporción entre el número de hojas marchitas y el número total de hojas por planta; existe una fuerte correlación positiva entre este índice y tolerancia a sequía (Pungulani et al., 2013).

Se ha reportado alta variabilidad entre genotipos en cuanto a potencial y estabilidad del rendimiento; algunos genotipos presentan buen comportamiento sin estrés y con estrés, mientras que otros presentan rendimientos buenos, bajo estrés hídrico, pero malos, sin estrés (Abayome y Abidoye, 2009). 
La reducción del rendimiento de semilla, en ensayos bajo sequía, también puede ser atribuido a la abscisión de estructuras reproductivas (Abayome y Abidoye, 2009; Ahmed y Suliman, 2010); genotipos de caupí de alto rendimientos de semilla, bajo condiciones de estrés hídrico, son importantes en la producción de este cultivo (Dadson et al., 2005).

Por otro lado, en líneas de caupí altamente tolerantes a sequía, la mayor cantidad de materia seca se distribuyó hacia las raíces y la menor proporción hacia la formación de vainas, al contrario de las líneas altamente susceptibles. Esta característica de las líneas tolerantes pareció ser muy ventajosa para colectar agua residual de las capas más profundas del suelo y con ello producir más en la estación seca (Watanabe et al., 1998).

\section{RELACIONES HÍDRICAS E INTERCAMBIO GASEOSO}

El potencial hídrico foliar es un indicador muy utilizado como medida del estado hídrico en las plantas, se mide, principalmente, antes del amanecer cuando la planta ha podido recuperar el agua perdida en el día y por tanto, está en equilibrio con el contenido de agua del suelo (Tardieu y Simonneau, 1998; Sellin, 1999).

El potencial hídrico se basa en el potencial químico del agua que es una expresión cuantitativa de la energía libre asociada con el agua o, más bien, el trabajo que se debe realizar para llevar una unidad de masa de agua, ligada al agua misma o al suelo hasta un estado de referencia cero, que es el agua pura (Taiz y Zeiger, 2010). Se han reportado valores aproximados de potenciales hídricos foliares letales en las plantas entre -1,4 a -6,0 MPa; en el fríjol caupí se han registrado entre $-1,5$ y $-2,5$ $\mathrm{MPa}$, lo que ha permitido clasificarla como una especie moderadamente tolerante al déficit de agua en el suelo (Boyer, 1978).
DoNascimento (2009), encontró que de un total de 20 genotipos de fríjol caupí, solo tres presentaron potenciales hídricos foliares mayores de -1,52 MPa, evidenciando con ello un mayor control de la apertura estomática, característica asociada a tolerancia a sequía en esta especie. En el mismo estudio reportó que uno de los genotipos registró un potencial hídrico de -2,05 $\mathrm{MPa}$; posiblemente el más bajo reportado en esta especie.

También se observó que el contenido hídrico relativo de la hoja está asociado con la disminución del contenido de humedad del suelo, presentándose amplia variación entre genotipos (Kumar et al., 2008; Pungulani et al., 2013). No obstante, para Singh y Reddy (2011) tal asociación no fue encontrada al evaluar 15 genotipos de caupí. Tampoco se ha encontrado correlación entre la conductancia estomática y el contenido hídrico relativo de la hoja, sugiriendo la existencia de una comunicación entre las raíces y los brotes, independiente del estado hídrico foliar (Agbicodo et al., 2009).

En general, el $\mathrm{CO}_{2}$ atmosférico se difunde a través de estomas hacia los espacios intercelulares, constituyendo la limitación estomática; luego, el $\mathrm{CO}_{2}$ se difunde a través del mesófilo (limitación mesofilar) en los sitios de carboxilación; esta, junto con la limitación bioquímica, constituye la limitación no estomática de la fotosíntesis, principalmente bajo estrés severo, que está relacionada con impedimentos metabólicos tales como limitaciones a la fosforilación, la regeneración de la 1,5 ribulosa bifosfato $(\mathrm{RuBp})$ y con la actividad de la rubisco (Singh y Reddy, 2011).

La limitación estomática utilizada para evitar la sequía podría explicarse, parcialmente, por un mecanismo eficiente que transfiere la información de las raíces a las hojas (Bates y Hall, 1981; Moreno, 2009). Se ha reportado que el cierre estomático como resultado de la disminución del contenido de humedad del suelo, mediado por cambios en el estado hídrico de la raíz, constitu- 
ye una respuesta al déficit hídrico en caupí y varía con el genotipo (Cruz de Carvalho et al., 1998; Anyia and Herzog, 2004; Hamidou et al., 2007; Singh y Reddy, 2011).

La disminución de la conductancia estomática, como consecuencia del cierre estomático, ocurre cuando el mesófilo comienza a sufrir deshidratación y está regulada por el ácido abscísico (Moreno, 2009). El contenido de ABA en la hoja se incrementa debido a su síntesis en las raíces y su transporte vía apoplasto con la corriente de transpiración hacia las células guarda, donde es liberado; también ocurre una redistribución del ABA desde los cloroplastos de las células del mesófilo (Zhang y Outlaw, 2001; Moreno, 2009).

El ABA causa pérdida de potasio, cloro o malato en las células guarda, lo que provoca disminución del volumen celular por la salida de agua del citoplasma, dando lugar al cierre estomático (Moreno, 2009; Taiz y Zeiger, 2010).

Se ha reportado que los genotipos que se marchitan temprano mantienen sus estomas abiertos durante la fase inicial del estrés, mientras que los que se marchitan tarde, los cierran (Agbicodo et al., 2009). A medida que avanza el estrés, los genotipos tolerantes a sequía sobreviven por el cierre estomático (Pungulani et al., 2013). Estudios realizados por Oliveira et al. (2005) muestran que la conductancia estomática es un indicador de estrés hídrico en fríjol caupí y reporta valores entre 14 y $165 \mathrm{mmol} \mathrm{m}^{-2} \mathrm{~s}^{-1} \mathrm{de}_{2} \mathrm{O}$, que varían según la fase fenológica y según el tiempo transcurrido desde la suspensión del suministro de agua. No obstante, al comparar genotipos sometidos a estrés hídrico, los valores más altos indican mayor tolerancia a sequía (DoNascimento, 2009).

Ensayos realizados en etapas tempranas, muestran que algunas plantas cierran sus estomas $y$ detienen el crecimiento, mientras que otras además de cerrar parcialmente sus estomas, hacen ajuste osmótico y continúan su crecimiento lentamente; las primeras evitan la sequía, mientras que las segundas la toleran, este parece ser el mecanismo más efectivo (Agbicodo et al., 2009).

En este mismo sentido, la reducción en la transpiración, regulada por el cierre estomático, es una respuesta común de las plantas al estrés por sequía que permite incrementar la eficiencia intrínseca en el uso del agua (A/gs) (Singh y Reddy, 2011). En efecto, la A/gs ha sido reconocida como una medida de la ganancia de carbono por unidad de agua perdida y es inversamente proporcional a la proporción de las concentraciones de $\mathrm{CO}_{2}$ intercelular y ambiental (Ci/Ca). Es evidente que una mayor eficiencia en el uso del agua y una mayor tasa fotosintética, junto a una mayor movilización de fotoasimilados hacia los órganos de cosecha, pueden mejorar el rendimiento de los cultivos bajo estrés hídrico (Condon et al., 2004; Parry et al., 2005; Singh y Reddy, 2011).

La eficiencia de transpiración (A/E) a nivel de la hoja, es más alta en genotipos tolerantes a sequía bajo condiciones de estrés hídrico y también puede ser medida por discriminación isotópica de carbono $\left(\Delta^{13} \mathrm{C}\right)$ en granos (Parry et al., 2005; Blum, 2009). Bajo estrés hídrico severo, varias líneas de caupí tolerantes a sequía, mostraron conductancia estomática y eficiencia de transpiración más altas que las sensibles (Belko et al., 2012).

El cierre parcial de estomas condujo también a un aumento de la temperatura de la zona meristemática de la hoja, pero permaneció constante a pesar del incremento del déficit de presión de vapor entre la hoja y el aire (Ben Haj Salah y Tardieu, 1997; Tardieu y Simonneau, 1998).

En relación con la limitación bioquímica, el transporte de electrones parece ser muy resistente a la inhibición bajo déficit hídrico, mientras que la fosforilación y el metabolismo fotosintéti- 
co parecen ser más sensibles a la deshidratación (Flexas et al., 2006). El rendimiento cuántico máximo fotoquímico no fue afectado por el estrés hídrico en Prunus salicina Lindh, indicativo de que la cadena de transporte de electrones es resistente a la deshidratación, de tal manera que la razón $\mathrm{F}_{\mathrm{V}} / \mathrm{FM}$ no es un indicador efectivo de estrés (Martinazzo et al., 2013).

La fosforilación puede ser sensible a los efectos tóxicos de la alta concentración de magnesio que acompaña la remoción de agua del cloroplasto en hojas deshidratadas (Kozlowski y Pallardy 1997; Taiz y Zeiger, 2010).

Por otro lado, cuando las plantas que pasan por un periodo de déficit hídrico son irrigadas, la tasa de fotosíntesis puede o no retornar a los niveles anteriores, dependiendo del material genético, severidad, duración de la sequía y la humedad relativa; las que consiguen recuperarse y vuelven a fotosintetizar como antes del estrés, poseen una mayor tolerancia al déficit hídrico (Kozlowski y Pallardy, 1997). No obstante, los mecanismos protectores que permiten mantener el aparato fotosintético del caupí, bajo estrés hídrico, aún no son bien conocidos (Anyia y Herzog, 2004; Singh y Reddy, 2011).

Se ha encontrado que los efectos perjudiciales de la sequía en el aparato fotosintético pueden durar días, semanas o hasta meses; por lo que la capacidad de las plantas estresadas de recuperar su capacidad fotosintética puede estar asociada a la capacidad de los estomas de reabrirse parcial o totalmente y a la síntesis de proteínas para superar el daño sufrido por el aparato fotosintético (Kozlowski y Pallardy, 1997; Pompelli et al., 2010).

Cruz de Carvalho et al. (1998) demostraron que la conductancia estomática (gs) y la tasa neta de asimilación (A, mmol m $\mathrm{m}^{-2}$ de $\mathrm{CO}_{2}$ medidas durante y después del tratamiento de estrés hídrico son parámetros confiables para identificar tolerancia a sequía en Phaseolus vulgaris y Vigna unguiculata.

\section{AJUSTE OSMÓTICO Y BIOMOLÉCULAS RELACIONADAS}

El ajuste osmótico consiste en una disminución del potencial hídrico en los tejidos vegetales, lo que permite la entrada de agua e impide la disminución en el turgor o en la actividad fotosintética; se origina a través de la biosíntesis de osmolitos orgánicos de bajo peso molecular y por la acumulación de iones, especialmente $\mathrm{K}^{+}$(Cushman, 2001; González Rodríguez et al., 2011).

La acumulación de iones durante el ajuste osmótico ocurre principalmente en la vacuola, mientras que en el citoplasma se acumulan solutos compatibles que no afectan negativamente la funcionalidad de macromoléculas celulares (Taiz y Zeiger 2010). Estos solutos son moléculas orgánicas de bajo peso molecular (osmolitos) como el aminoácido prolina, alcoholes-azúcar como el sorbitol y el manitol y una amina cuaternaria llamada glicina-betaína (Rodríguez, 2006; Moreno, 2009). Diferentes tipos de organismos como plantas, bacterias, hongos y animales presentan osmolitos compatibles que se caracterizan por no alterar la estructura y función de las macromoléculas, cuando se acumulan en altas concentraciones (Taiz y Zeiger, 2010).

La acumulación de osmolitos compatibles también tiene como consecuencia la osmoprotección, que está dada por la capacidad estabilizadora de algunos de estos solutos sobre macromoléculas como las proteínas y los sistemas de membrana celulares. Moléculas como prolina y glicina-betaína, mediante la formación de puentes de hidrógeno, pueden inducir la formación de puentes de hidrógeno alrededor de proteínas, preservando su estado original (KaviKishor et al., 2005). 
En general, la osmorregulación permite a las plantas tolerar condiciones de escasez de agua y salinidad elevadas, e involucra mecanismos adaptativos que evitan la disminución de la fotosíntesis, las alteraciones en la traslocación y la distribución de fotoasimilados, y la pérdida de rendimiento (Rodríguez, 2006).

En plantas de caupí sometidas a déficit hídrico, se reportaron significativos incrementos de prolina que propiciaron ajuste osmótico (Lobato et al., 2008). No obstante, para algunos autores, el aumento en el contenido de prolina, no está claro si contribuye al ajuste osmótico, dado que su papel en caupí ha sido reportado como síntoma de lesiones en las hojas (Bates y Hall, 1981; Singh y Reddy, 2011).

También se ha reportado en caupí, bajo estrés hídrico, incrementos de carbohidratos y aminoácidos totales, y disminución de la cantidad de proteína total (Costa et al., 2008; Farouk y Amani, 2012). Además, reducciones significativas en clorofila total (Lobato et al., 2009; Abass y Mohamed, 2011; Farouk y Amani, 2012), y carotenoides, considerados típicos indicadores de estrés oxidativo atribuidos a la fotooxidación de pigmentos y a la degradación o deficiencia en la síntesis de clorofilas, conducentes a minimizar la absorción de luz por los cloroplastos (Singh y Reddy, 2011). Los mismos autores reportaron un aumento en el contenido de cera epicuticular que podría contribuir a la disminución de la transpiración.

\section{PROCESOS DE DETOXIFICACIÓN DE ESPECIES REACTIVAS DE OXÍGENO}

Las especies reactivas de oxígeno, tales como peróxido de hidrógeno $\left(\mathrm{H}_{2} \mathrm{O}_{2}\right)$, radical hidroxilo $\left(\mathrm{OH}^{-}\right)$y anión superóxido $\left(\mathrm{O}_{2}^{-}\right)$, aumentan como producto de la disipación del exceso de energía, lo que generalmente ocurre junto con la intensificación de las limitaciones estomáticas y no estomáticas, frecuentes en épocas secas; simul- táneamente, se activan mecanismos bioquímicos que las contrarrestan (Tausz et al., 2004). En condiciones de estrés hídrico estos mecanismos bioquímicos pueden contribuir al mantenimiento de diversos procesos fisiológicos tales como la apertura estomática, la fotosíntesis y la expansión celular (Lawlor y Cornic, 2002).

Entre las enzimas del sistema antioxidante involucradas en procesos de detoxificación de ROS están la catalasa CAT, la ascorbato peroxidasa APX, la superóxido dismutasa SOD y glutatión reductasa GR, además de aldehído malónico MDA (Pompelli et al., 2010). Las enzimas glutatión reductasa y ascorbato peroxidasa desempeñan un papel clave en fríjol caupí (Agbicodo et al., 2009).

El incremento de la actividad de las enzimas antioxidantes en plantas sometidas a estrés por sequía, podría contribuir a su capacidad para sobrevivir en ambientes áridos adversos (Pompelli et al., 2010).

Se ha indicado que el estrés por sequía conduce a diferentes niveles de expresión de GR en caupí y fríjol común, según sus grados de tolerancia a sequía (Torres-Franklin et al., 2008) y, se ha reportado la expresión de un gen relacionado con la enzima homoglutation sintetasa (hGSHS) en respuesta al estrés por sequía en esta especie (Cruz de Carvalho et al., 2010).

Por otro lado, las plantas tolerantes a sequía mantienen la composición lipídica de las membranas celulares, como resultado de mecanismos antioxidantes eficientes y control de actividades hidrolíticas (Matos et al., 2008). Los ácidos grasos poliinsaturados y específicamente el ácido linolénico se incrementan en las hojas de plantas de caupí tolerantes a sequía, mientras que en las sensibles disminuyen (Torres-Franklin et al., 2009).

Se ha indicado que el contenido de MDA, uno de los últimos productos de la peroxidación de lípidos por efecto del daño provocado por los ra- 
dicales libres, es un indicador de daño oxidativo (Pompelli et al., 2010).

\section{ACTIVIDAD MOLECULAR}

Además de las respuestas fisiológicas y bioquímicas inducidas por el estrés debido a sequía, las plantas también responden y se adaptan a nivel celular y molecular, y muchos genes son inducidos o represados bajo tal condición de estrés; recientemente han sido identificados varios de los genes inducidos por sequía, utilizando el análisis de micromatrices (transcriptómica), tecnología que emplea ADNc u oligonucleótidos para analizar la expresión de genes de plantas expuestas a sequía (Ansari y Lin, 2010).

Los productos de los genes inducidos por sequía, identificados con esta técnica, incluyen proteínas chaperonas, proteínas LEA (late embryogenesis abundant), osmotina, enzimas clave para la biosíntesis de osmolitos, proteínas canales de agua, azúcar y transportadores de prolina, enzimas detoxificantes y varias proteasas; también se producen proteínas reguladoras, tales como kinasas, fosfatasas, enzimas involucradas en el metabolismo de fosfolípidos y otras como la calmodulina (Ansari y Lin, 2010).

Por otro lado, con el desarrollo rápido de la biología molecular, los marcadores moleculares han sido ampliamente usados en el mejoramiento genético de varios cultivos, incluyendo el caupí (Tan et al., 2012). Las técnicas moleculares han permitido importantes logros en mejoramiento genético, tales como análisis de diversidad genética, construcción de mapas de ligamientos genéticos y mapeo de OTL (Muchero et al., 2009).

Para tales propósitos se han aplicado marcadores RAPD, AFLP y SSR (de uso más frecuente), mientras que SNP aún no han sido usado en análisis de diversidad genética en esta especie
(Tan et al., 2012; Patil, 2013). Sin embargo, los SNP han ganado mucho interés por la comunidad mejoradora de plantas (Ganal et al., 2009) y recientemente se reportó que una colección mundial de caupí, compuesta por 422 variedades locales, procedentes de 56 países, fue genotipada con más de 1.200 SNP, encontrándose dos pools de genes; el pool 1, mayoritariamente distribuido al occidente de África y, el pool 2, más relacionado con el oriente de África y parece ser que las accesiones de América están más estrechamente ligadas al pool 2 (Huynh et al., 2013).

No obstante, la aplicación de estos marcadores está aún en etapa de exploración, por lo que es necesario profundizar en estas técnicas para acelerar el mejoramiento asistido por marcadores moleculares. El mapeo de genes candidatos ofrece una solución alternativa para identificar los factores determinantes de características subyacentes a QTL de interés (Muchero et al., 2010).

Al usar estos marcadores moleculares, para la extracción de ADN de alta calidad se recomienda aislarlo de hojas jóvenes de plántulas de caupí, lo cual resulta de bajo costo, rápido, de alta pureza, integro, y funcional en la digestión de la endonucleasa de restricción y en el análisis posterior basado en PCR (Tan et al., 2013).

Con estas técnicas se pretende aislar e identificar las principales vías genéticas involucradas en la respuesta a sequía en caupí. Al respecto, se han encontrado altas correlaciones entre el mantenimiento del color verde del tallo, sobrevivencia, recuperación de materia seca y senescencia en campo, que sugieren un proceso basado en la senescencia de tejidos presente en la tolerancia a sequía en plántulas de caupí; afirmación soportada en ADNc asociado a senescencia, aislado del caupí sometido a estrés hídrico (Muchero et al., 2008).

Por otro lado, OTL (locus de un carácter cuantitativo) asociados con tolerancia a la sequía en 
estado de plántula y en la madurez, en líneas endogámicas de caupí, fueron identificados por Muchero et al. (2009) y Tan et al. (2012) y encontraron alta correlación entre el estado verde del tallo a la madurez y el peso seco de la planta, por lo que la selección fenotípica representa un mecanismo efectivo para identificar plantas con tolerancia a sequía como ha sucedido en sorgo (Subudhi et al., 2000). Posteriormente, se identificaron 5 loci que evidenciaron efecto pleiotrópico entre la permanencia verde de la planta y la senescencia tardía, biomasa y rendimiento de grano (Muchero et al., 2013).

Más recientemente, se ha encontrado que modificaciones epigenéticas, por metilación del ADN, y mecanismos basados en cromatina y pequeños ARN, pueden contribuir, separadamente o en conjunto con los fenotipos, a regular la expresión de genes en respuesta al efecto del estrés (Grativol et al., 2012).

\section{CONCLUSIONES}

Son muchos los aspectos asociados a las respuestas al déficit hídrico en las plantas y particularmente al fríjol caupí. Los estudios enfocados a seleccionar genotipos con mayores grados de tolerancia al déficit hídrico, se han dirigido hacia cinco grandes aspectos interrelacionados: las modificaciones del crecimiento y desarrollo de los órganos de la planta, la dinámica de las relaciones hídricas e intercambio gaseoso, los procesos bioquímicos que incluyen las limitaciones bioquímicas asociadas a proteínas y otros procesos, el ajuste osmótico, los procesos de detoxificación de especies reactivas de oxígeno por la actividad de enzimas antioxidantes y la actividad molecular. Este último aspecto pretende identificar vías genéticas asociadas a características morfológicas y fisiológicas que han desarrollado las plantas a lo largo de su proceso evolutivo.

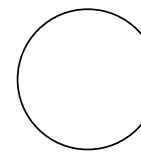

Abass, S.M. y H.I. Mohamed. 2011. Alleviation of adverse effects of drought stress on common bean (Phaseolus vulgaris L.) by exogenous application of hydrogen peroxide. Bangladesh J. Bot. 41(1), 75-83.

Abayome, Y.A. y T.O. Abidoye. 2009. Evaluation of cowpea genotypes for soil moisture stress tolerance under screen house conditions. African J. Plant Sci. 3 (10), 229-237.

Agbicodo, E.M., C.A. Fatokun, S. Muranaka, R.G.F. Visser y C.G. Linden Van Der. 2009. Breeding drought tolerant cowpea: constraints, accomplishments, and future prospects. Euphytica 167, 353-370.

Ahmed, F.E. y A.S.H. Suliman. 2010. Effect of water stress applied at different stages of growth on seed yield and water-use efficiency of cowpea. Agric. Biol. J. N. Am. 1(4), 534-540.

Ansari, M.I. y T.P. Lin. 2010. Molecular Analysis of Dehydration in Plants. Intl. Res. J. Plant Sci. 1(2), 21-25.

\section{REFERENCIAS BIBLIOGRÁFICAS}

Anyia, A.O. y H. Herzog. 2004. Genotypic variability in drought performance and recovery in cowpea under controlled environment. J. Agron. Crop Sci. 190 (2), 151-159.

Bates, L.M. y A.E. Hall. 1981. Stomatal closure with soil water depletion not associated with changes in bulk leaf water status. Oecologia. 50, 62-65.

Beebe, S.E., I.M. Rao, M.W. Blair y J.A. Acosta-Gallego. 2013. Phenotyping common beans for adaptation to drought. Front. Physiol. 4, 35.

Belko, N., M. Zaman-Allah, N. Cisse, N.N. Diop, G. Zombre, J.D. Ehlers y V. Vadez. 2012. Lower soil moisture threshold for transpiration decline under water deficit correlates with lower canopy conductance and higher transpiration efficiency in drought-tolerant cowpea. Funct. Plant Biol. 39(4), 306-322.

Ben-Haj-Salah, H. y F. Tardieu. 1997. Control of leaf expansion rate of droughted maize plants under 
fluctuating evaporative demand. A superposition of hydraulic and chemical messages? Plant Physiol. 114, 893-900.

Blum, A. 2009. Effective use of water (EUW) and not water-use efficiency (WUE) is the target of crop yield improvement under drought stress. Field Crop. Res. 112 (2009), 119- 123.

Boyer, J.S. 1978. Chapter 4: Water deficits and photosynthesis. pp. 154-191. En: Kozlowski, T. T. (ed.). Water deficits and plant growth. Academic Press, N.Y.

Condon, A.G., R.A Richards, G.J. Rebetzke y G. D. Farquhar. 2004. Breeding for high water-use efficiency. J. Exp. Bot. 55 (407), 2447-2460.

Costa, R.C.L., A.K.S. Lobato, C.F. Oliveira Neto, P.S.P. Maia, G.A.R. Alves y H.D. Laughinghouse. 2008. Biochemical and physiological responses in two Vigna unguiculata (L.) Walp. Cultivars under water stress. J. Agron. 7, 98-101.

Cruz de Carvalho M.H., D. Laffray y P. Louguet. 1998. Comparison of the physiological responses of Phaseolus vulgaris and Vigna unguiculata cultivars when submitted to drought conditions. Environ. Exp. Bot. 40,197-207.

Cruz de Carvalho, M.H., H. Brunet, I. Kranner, A.D. Arcy-Lammeta, Y. Zuily-Fodil y D. Cantour-Ansel. 2010. Homoglutathione synthetase and glutathione synthetase in drought-stressed cowpea leaves: Expression patterns and accumulation of low-molecular-weight thiols. J. Plant Physiol. 167,480-487.

Cushman, J.C. 2001. Osmoregulation in plants: implications for agriculture. Am. Zool. 4, 758-769.

Dadson, R.B., F.M. Hashem, I. Javaid, J. Joshi, A.L. Allen y T.E. Devine. 2005. Effect of water stress on the yield of cowpea (Vigna unguiculata L. Walp.) genotypes in the Delmarva region of the United States. J. Agron. Crop Sci. 191, 210-217.

DoNascimento, S.P. 2009. Efeito do deficit hídrico em feijão-caupi para entificação genótipos com tolerância à seca. Tesis de mestre em agronomía. Agricultural Science Center, Universidad Federal do Piauí, Teresina, Brasil.

Farouk, S. y A.R. Amany. 2012. Improving growth and yield of cowpea by foliar application of chitosan under water stress. Egypt. J. Biol. 14, 14-26.

Flexas, J., J. Bota, J. Galmés, H. Medrano y M. RibasCarbó. 2006. Keeping a positive carbon balance under adverse conditions: responses of photosynthesis and respiration to water stress. Physiol. Plant. 127(3), 343-352.
Ganal, M.W., T. Altman y M.S. Roder. 2009. SNP identification in crop plants. Curr. Opin. Plant Biol.12, 211-217.

González-Rodríguez, H., I. Cantú-Silva, R.G. RamírezLozano, M.V. Gómez-Meza, M. Pando-Moreno y J.M. López-Hernández. 2011. Potencial hídrico xilemático en cuatro especies arbustivas nativas del noreste de México. Revista Chapingo Serie Ciencias Forestales y del Ambiente 17, 97-109.

Grativol, C., A.S. Hemerly y P.C.G Gomes Ferreira. 2012. Genetic and epigenetic regulation of stress responses in natural plant populations. Biochim. Biophys. Acta 1819, 176-185

Hall, A. 2004. Breeding for adaptation to drought and heat in cowpea. Europ. J. Agron. 21, 447-454.

Hall, A.E.; N. Cisse, S. Thiaw, H.O.A Elawad, J.D. Ehlers, A.M. Ismail, R. Fery, P.A. Roberts, L.W. Kitch, L.L. Murdock, O. Boukar, R.D. Phillips y K.H Mcwatters. 2003. Development of cowpea cultivars and germplasm by the Bean/Cowpea CRSP. Field Crop Res. 82, 103-134.

Hamidou, F., G. Zombre, O. Diouf, N. Diop, S. Guinko, and S. Braconnier. 2007. Physiological, biochemical and agromorphological responses of five cowpea genotypes (Vigna unguiculata (L.) Walp.) to water deficit under glasshouse conditions. Biotechnol. Agron. Soc. Environ. 11(3), 225-234.

Huynh, B-L., T.J. Close, P.A. Roberts, Z. Hu, S. Wanamaker, R.M.R Mitchell R. Chiulele, N. Cissé, A. David, S. Hearne, C. Fatokun, N.N. Diop y J.D. Ehlersthe. 2013. Gene pools and the genetic architecture of domesticated cowpea. Plant Genet. 6(2), 1-8.

Ichi, J.O., H.E. Igbadun, S. Miko and A.M. Samndi. 2013. Growth and yield response of selected cowpea (Vigna unguiculata L. Walp.) variety to irrigation interval and sowing date. The Pacific J. Sci. Techn. 14 (1), 453-463.

Ishiyaku, M.F. and H. Aliyu. 2013. field evaluation of cowpea genotypes for drought tolerance and Striga resistence in the dry savanna of the North-West Nigeria. Int. J. Plant Breed. Genet. 7(1), 47-56.

Kavi-Kishor, P.B., Z. Hong, G.H. Miao, C.A.A Hu y D.P.S. Verma. 1995. Overexpression of D1-pyrroline-5-carboxylate synthetase increases proline production and confers osmotolerance in transgenic plants. Plant Physiol. 108, 1387-1394.

Kozlowski, T.T. y S.G. Pallardy . 1997. Physiology of woody plants. $2^{\text {nd }}$ ed. Academic Press. Academic Press. San Diego, CA. pp. 147-153. 
Kumar, A., K.D. Sharma y D. Kumar. 2008. Traits for screening and selection of cowpea genotypes for drought tolerance at early stages of breeding. J. Agric. Rur. Develop. Trop. Subtrop. 109(2), 191-199.

Lawlor, D.W. y G. Cornic. 2002. Photosynthetic carbon assimilation and associated metabolism in relation to water deficits in higher plants. Plant Cell Environ. $25,275-294$

Lobato, A.K.S., C.F. Oliveira Neto, R.C.L. Costa, B.G. Santos-Filho, F.J.R. Cruz y H.D. Laughinghouse. 2008. Biochemical and physiological behavior of Vigna unguiculata (L.) Walp. under water stress during the vegetative phase. Asian J. Plant Sci. 7, 44-49.

Lobato, A.K.S, R.C.L. Costa, C.F.O. Neto, B.G.S. Filho, G.A.R. Alves, J.M.N. Freitas, F.J.R. Cruz, C.A. Marochio y G.K. Coimbra. 2009. Responses of the pigments and carbon metabolism in Vigna unguiculata cultivars submitted to water deficit. Res. J. Biol. Sci. 4, 593-598.

Martinazzo, E.G., A.T. Perboni, P.V. Deoliveira, V.J. Bianchi y M.A. Bacarin. 2013. Photosynthetic activity in Japanese plum under water deficit and flooding. Ciência Rural 43(1), 35-41.

Matos, A.R., Gigon, A. Laffray, D. Pêtres, S. Zuily-Fodil y A.T. Pham-Thi. 2008. Effects of progressive drought stress on the expression of patatin-like lipid acyl hydrolase genes in Arabidopsis leaves. Physiol. Plant. 134(1), 110-120.

Matsui, T. y B. Singh. 2003. Root characteristics in cowpea related to drought tolerance at the seedling stage. Exp. Agric. 29, 29-38.

Moreno, L.P. 2009. Respuesta de las plantas al estrés por déficit hídrico. Una revisión. Agron. Colomb. 27(2), 179-191.

Muchero, W., J.D. Ehlers y P.A. Roberts. 2008. Seedling stage drought-induced phenotypes and droughtresponsive genes in diverse cowpea genotypes. Crop Sci. 48, 541-552.

Muchero, W., J.D. Ehlers, T. Close y P. Roberts. 2009. Mapping QTL for drought stress-induced premature senescence and maturity in cowpea (Vigna unguiculata (L.) Walp.). Theor. Appl. Genet. 118, 849-863.

Muchero, W., J.D. Ehlers y P.A. Roberts. 2010. Restriction site polymorphism-based candidate gene mapping for seedling drought tolerance in cowpea Vigna unguiculata (L.) Walp. Theor. Appl. Genet. 120, 509-518.

Muchero, W., P.A. Roberts, N.N. Diop, I. Drabo, N. Cisse, T.J. Close, S. Muranka, O. Boukar y J.D. Ehlers.
2013. Genetic architecture of delayed senescence, biomass, and grain yield under drought stress in cowpea. PloS ONE 8(7), e70041.

Nelson, C., M. Rosegrant, J. Koo, R. Robertson, T. Sulser, T. Zhu, C. Ringler, S. Msangi, A. Palazo, M. Batka, M. Magalhaes, R. Valmonte-santos, M. Ewing y D. Lee. 2009. Cambio climático: el impacto en la agricultura y los costos de adaptación. Instituto Internacional de Investigación sobre Políticas Alimentarias IPFRI. En: http://www.fao.org; consulta: agosto de 2010

Okon, I.E. 2013. Effect of water stress on some growth aspect of two varieties of cowpea, Vigna unguiculata L. Walp. Fabaceae. Bullet. Environ. Pharmacol. Life Sci. 2(5), 69-74.

Oliveira, A. D., E.J. Fernandes y T.J.D. Rodrigues. 2005. Condutância estomática como indicador de estresse hídrico em Feijão. Engenharia Agrícola 25(1), 86-95.

Parry, M.A.J., J. Flexas y H. Medrano. 2005. Prospects for crop production under drought: research priorities and future directions. Ann. Appl. Biol. 147 211-226

Patil, D.M., N.B. Sawardekar, S.G. Gokhale, S.S. Bhave, S.A. Sawant, K.A. Sawantdesai, S.N. Lipne, S.N Sabale y S.N. Joshi. 2013. Genetic diversity analysis in cowpea (Vigna unguiculata L. Walp.) by using RAPD markers. Intl. J. Innov. Biotechnol. Biochem. $1(1), 15-23$

Polanía, J.A., I.M. Rao, S. Mejía, S.E. Beebe y C. Cajiao. 2012. Características morfo-fisiológicas del fríjol común (Phaseolus vulgaris L.) relacionadas con la adaptación a sequía. Acta Agron. 61(3), 197-206.

Pompelli, M., R. Barata, H. Vitorino, E. Gonçalves, E. Rolim, M. Santos, J. Almeida, V. Ferreira, E. Lemos y L. Endres. 2010. Photosynthesis, photoprotection and antioxidant activity of purging nut under drought deficit and recovery. Biom. Bioenergy 34 (8), 1207-1215

Pungulani, L.L.M., J.P Millner, W.M. Williams y M. Banda. 2013. Improvent of leaf wilting scoring in cowpea (Vigna Sinensis (L.) Walp.): From qualitative scale to quantitative index. Aust. J. Crop Sci. 7 (9), 1262-1269

Qasem, J.R. y K.N. Biftu. 2010. Growth analysis and responses of cowpea (Vigna Sinensis (L.) Savi ExHassk. and redroot pigweed (Amaranthus retroflexus L.), grown in pure and mixed stands, to density and water stresses. The Open Hortic. J. 2010(3), 21-30.

Reis, G.G. y A.E. Hall. 1987. Relações hídricas e atividade do sistema radIcular em Eucalyptus camaldulensis Dehn. em condições de campo. Revista Árvore 11(1), 43-55 
Rodríguez, L. 2006. Implicaciones fisiológicas de la osmorregulación en plantas. Agron. Colomb. 24(1), 28-37.

Sellin A. 1999. Does pre-dawn water potential reflect conditions of equilibrium in plants and soil water status? Acta Oecol. 20 (1), 51-59.

Singh, S.K. y K.R. Reddy. 2011. Regulation of photosynthesis, fluorescence, stomatal conductance and water-use efficiency of cowpea (Vigna unguiculata (L.) Walp.) under drought. J. Photochem. Photobiol. B: Biol. 105, 40-50.

Subudhi, P.K., D.T. Rosenow y H.T. Nguyen. 2000. Quantitative trait loci for the stay green trait in sorghum (Sorghum bicolor L. Moench): consistency across genetic backgrounds and environments. Theor. Appl. Genetics. 101,733-741.

Taiz, L. y E. Zeiger. 2010. Plant physiology. $5^{\text {th }}$ ed. Sinauer Associates, Inc. Redwood City, CA. pp. 644-645.

Tan, H., M. Tie, Q. Luo, Y. Zhu, J. Lai y H. Li. 2012 A review of molecular markers applied in cowpea (Vigna unguiculata L. Walp.) breeding J. Life Sci. 6, 1190-1199

Tan, H., H. Huang, M. Tie, J. Ma y H. Li. 2013. Comparative analysis of six DNA extraction methods in cowpea (Vigna unguiculata L. Walp). J. Agr. Sci. 5(7), 82-90.

Tardieu, F. y T. Simonneau. 1998. Variability of species among stomatal control under fluctuating soil water status and evaporative demand: modeling isohydric and anisohydric behaviours. J. Exp. Bot. 49, 419-432.

Tausz M., H.S. Ircelj and D. Grill. 2004. The glutathione system as a stress marker in plant ecophysiology: is a stress-response concept valid? J. Exp. Bot. 55 (404), 1955-1962.
Timko, M.P., P.J. Rushton, T.W. Laudeman, M.T. Bokowiec, E. Chipumuro, F. Cheung, C.D. Town y X. Chen. 2008. Sequencing and analysis of the generich space of cowpea. BMC Genomics 9, 103 .

Torres-Franklin, M.L, D. Contour-Ansel, Y. Zuily-Fodil y A.T. Pham-Thi. 2008. Molecular cloning of glutathione reductase cDNAs and analysis of GRgene expression in cowpea and common bean leaves during recovery from a moderate drought stress. J. Plant Physiol. 165, 514-21.

Torres-Franklin, M.L., A. Repellina, V.B. Huynh, A.D. Arcy-Lammeta, Y. Zully-Fodil y T. Pham-Thia. 2009. Omega-3 fatty acid desaturase (FAD3, FAD7, FAD8) gene expression and linolenic acid content in cowpea leaves submitted to drought and after rehydration. Environ. Exp. Bot. 65, 162-169.

Van Duivenbooden, N., S. Abdoussalam y A.B. Mohamed. 2002. Impact of climate change on agricultural production in the Sahel-Part 2.Case study for groundnut and cowpea in Niger. Climatic Change 54(3), 349-368

Wahid, A., S. Gelani, M. Ashraf y M.R. Foolad. 2007. Heat tolerance in plants: An overview. Environ. Exp. Bot. 61(3), 199-223.

Watanabe, I., I. Tsukuba y T. Terao. 1998. Drought tolerance of cowpea (Vigna unguiculata (L.) Walp.), 2: Field trial in the dry season of Sudan savanna and dry matter production of potted plants under water-stress. J. Int. Res. Cent. Agr. Sci. 6, 29-37.

Zhang S.Q. y W.H. Outlaw. 2001. Abscisic acid introduced into the transpiration stream accumulates in the guard cell apoplasto and causes stomatal closure. Plant Cell Environ. 24, 1045-1054. 